\title{
Tratamento conservador para rotura hepática em Síndrome HELLP: um relato de caso
}

\author{
Conservative treatment for hepatic rupture in HELLP Syndrome: case report \\ Tratamiento conservador para la rotura hepática en el Síndrome HELLP: presentación de \\ caso
}

Verônica Tavares Ribeiro ${ }^{1 *}$, Theófilo Mendes do Bom Conselho ${ }^{1}$, Lucas Maciel Dutra1, Rhavi Maletta Noleto ${ }^{1}$, Guilherme Tofane Maia VilasBoas ${ }^{1}$, Henrique Martins Damini', Laura Amaral de Lara Resende ${ }^{1}$, Isabela Hermont Duarte ${ }^{2}$, Luiza Liboreiro Motta Ferrari ${ }^{3}$, Luiza Gontijo Araujo Lamounier ${ }^{1}$.

\section{RESUMO}

Objetivo: O presente estudo busca elucidar o caso de uma paciente admitida no serviço de alto risco em ginecologia e obstetrícia em um hospital em uma cidade da região metropolitana de Belo Horizonte, sendo realizado o diagnóstico de Síndrome HELLP complicada com rotura hepática e conduzido com tratamento conservador. Detalhamento do caso: Primigesta de 19 anos com síndrome HELLP em idade gestacional de 37 semanas, necessitando de parto imediato por via abdominal, que resultou em morte fetal. O diagnóstico de hematoma hepático subcapsular roto foi estabelecido no pós-parto, mediante tomografia computadorizada de abdome, após piora laboratorial. $O$ quadro foi manejado com plasmaférese e pulsoterapia de metilprednisolona. A paciente se recuperou completamente após um mês de hospitalização. Considerações finais: É imprescindível manter estrita vigilância nos parâmetros clínico-laboratoriais das pacientes diagnosticadas com Síndrome HELLP, devido a gravidade do quadro as potenciais complicações. Apesar da escassez de estudos referentes a Síndrome HELLP complicada por rotura hepática, o tratamento não cirúrgico pode ser benéfico na condução dessas pacientes, proporcionando desfechos favoráveis quando bem indicado.

Palavras-chave: Plasmaférese, Síndrome HELLP, Ruptura hepática.

\begin{abstract}
Objective: The present study seeks to elucidate the case of a patient admitted to the high-risk service in gynecology and obstetrics at a hospital in a city in the metropolitan region of Belo Horizonte, with a diagnosis of HELLP syndrome complicated with hepatic rupture and conducted with conservative treatment. Case details: A 19-year-old primigravida with HELLP at 37 weeks of gestation, requiring urgent delivery via cesarean section and resulting in fetal death. The diagnosis of ruptured subcapsular liver hematoma was established in postpartum with abdominal CT imaging, after worsening of laboratory parameters. She was managed with plasmapheresis and methylprednisolone pulse therapy and made a full recovery after a onemonth hospitalization. Final considerations: Early diagnosis and the monitoring of clinical e laboratory parameters are of utmost importance in these patients, given the severity and possible complications of the
\end{abstract}

\footnotetext{
${ }^{1}$ Faculdade Ciências Médicas de Minas Gerais (FCMMG). Belo Horizonte - MG.

*E-mail: vetavares05@gmail.com

${ }^{2}$ Faculdade de Minas (Faminas). Belo Horizonte - MG.

${ }^{3}$ Hospital Vila da Serra. Nova Lima - MG.
}

SUBMETIDO EM: 8/2020

ACEITO EM: 9/2020

PUBLICADO EM: 10/2020 
disease. Even if literature on the conservative management of ruptured subcapsular liver hematoma in patients with HELLP, this might potentially be the preferred alternative, if it is adequately indicated.

Key words: Plasmapheresis, HELLP syndrome, Hepatic rupture.

\section{RESUMEN}

Objetivo: El presente estudio busca dilucidar el caso de una paciente ingresada en el servicio de alto riesgo de ginecología y obstetricia de un hospital de una ciudad de la región metropolitana de Belo Horizonte, con el diagnóstico de síndrome HELLP complicado con rotura hepática y realizado con tratamiento conservador. Detalles del caso: Una primigrávida de 19 años con HELLP a las 37 semanas de gestación, que requirió un parto urgente por cesárea y resultó en muerte fetal. El diagnóstico de hematoma hepático subcapsular roto se estableció en el posparto a través de TC abdominal, tras empeoramiento de los parámetros analíticos. La condición se manejó con plasmaféresis y terapia de pulsos de metilprednisolona. El paciente se recuperó completamente después de un mes de hospitalización. Consideraciones finales: El diagnóstico precoz y el seguimiento de los parámetros clínicos y de laboratorio son de suma importancia en estos pacientes, dada la gravedad y las posibles complicaciones de la enfermedad. Incluso si existe literatura sobre el tratamiento conservador del hematoma hepático subcapsular roto en pacientes con HELLP, esta podría ser potencialmente la alternativa preferida, si está adecuadamente indicada.

Palabras clave: Plasmaféresis, Síndrome HELLP, Rotura hepática.

\section{INTRODUÇÃO}

A Síndrome HELLP é uma condição específica da gravidez, podendo ou não estar associada a préeclâmpsia ou eclâmpsia (TROJA A, et al., 2015). Sua fisiopatogenia permanece pouco conhecida, mas acredita-se que tem origens semelhantes às da pré-eclâmpsia, sendo uma rejeição imunológica aguda que ocasiona invasão inadequada do citotrofoblasto, remodelando as artérias uterinas e artérias em espiral, de modo a reduzir a pressão de perfusão útero-placentária com consequente liberação de citocinas, mediadores inflamatórios, fatores antiangiogênicos e outras substâncias na circulação materna. HELLP é o acrônimo de hemólise (Hemolysis), elevação de enzimas hepáticas (Elevated Liver enzymes) e plaquetopenia (Low Platelets) (BRILHANTE AVM, et al., 2010). Tem como principal fator de risco o histórico de pré eclâmpsia e síndrome HELLP em gestações prévias. Atinge de 0,5 a $0,9 \%$ de todas as gestações e de 4 a $18,9 \%$ das pacientes com pré-eclâmpsia (TROJA A, et al., 2015).

A incidência da Síndrome HELLP se dá, principalmente, da 27 a 37 semanas de gestação, podendo ocorrer também de 24 a 48 horas após o parto (MAEJI IL, et al., 2019; TROJA A, et al., 2015). Ressalta-se sua grande importância haja vista as altas taxas de morbidade e mortalidade, sendo a última perinatal fetal de 10 a $60 \%$ e materna de 1,5 a 5\% (POLLO-FLORES P, et al., 2015). Ademais, a HELLP também pode evoluir com complicações, tais quais: edema pulmonar agudo; insuficiência renal; coagulação intravascular disseminada (CIVD); hemorragia do sistema nervoso central; hematoma hepático e rotura hepática (TROJA A, et al., 2015).

No que tange ao hematoma subcapsular hepático e sua ruptura constata-se que são complicações raras, ocorrendo em cerca de 1 a $2 \%$ dos casos, com taxas de letalidade que chegam a até $90 \%$ (BRILHANTE AVM, et al., 2010).

O quadro é marcado por progressão da lesão hepática gerada pela síndrome, ocasionando notória elevação dos níveis séricos de aminotransferases, LDH e bilirrubinas. A apresentação clínica é caracterizada por intensa dor no hipocôndrio direito, podendo irradiar para o membro superior direito, ocasionar náuseas, vômitos e sinais de choque hemorrágico (MACHADO LG, et al., 2010). Quando a paciente se encontra estável hemodinamicamente, o diagnóstico é realizado por exames de imagem, sendo a tomografia computadorizada o exame de escolha (MAEJI IL, et al., 2019). 
O presente estudo busca elucidar o caso de uma paciente admitida no serviço de alto risco em ginecologia e obstetrícia em um hospital de uma cidade da região metropolitana de Belo Horizonte, sendo realizado o diagnóstico de Síndrome HELLP complicada com rotura hepática e conduzido com tratamento conservador.

\section{DETALHAMENTO DO CASO}

Paciente SLCS, 19 anos, primigesta com 37 semanas de Idade Gestacional, foi admitida no serviço de alto risco em Ginecologia e Obstetrícia do Hospital com sinais de Crescimento Intrauterino Restrito (CIUR), evidenciado ao ultrassom abdominal. Nesse exame, realizou-se o estudo com doppler fetal - sem alterações - e doppler materno, cuja avaliação evidenciou aumento de resistência em artérias uterinas. Procedeu-se à internação da paciente com prescrição de corticoterapia.

Após 48 horas de internação, evoluiu com fortes contrações, cefaléia frontal, vômitos e dor severa em quadrante superior do abdome. Nesse momento, a pressão arterial aferida foi de $170 \times 100 \mathrm{mmHg}$. Optou-se pela realização de cardiotocografia (CTG), que evidenciou sofrimento fetal agudo, sendo indicada cesariana de urgência, visto quadro materno e fetal. $O$ parto cirúrgico foi realizado e teve como complicações: descolamento prematuro de placenta, hipotonia uterina e, por fim, óbito fetal. Após o procedimento, a paciente foi encaminhada no mesmo dia ao Centro de Terapia Intensiva (CTI). Os exames laboratoriais na admissão revelaram aumento de transaminases hepáticas (TGO $738 \mathrm{U} / \mathrm{L} / \mathrm{TGP} 639 \mathrm{U} / \mathrm{L}$ ) e LDH (4500 mg/dL), anemia (Hb 9,5 g/dL), plaquetopenia $\left(97000 / \mathrm{mm}^{3}\right)$ e leucocitose $\left(32070 / \mathrm{mm} /{ }^{3}\right)$.

A tomografia computadorizada (TC) de abdome apresentou fígado com dimensões aumentadas, parênquima com alterações perfusionais, e imagem sugestiva de hematoma subcapsular (Figura 1). Paciente evoluiu ao longo da internação em estado grave, com elevações ainda mais pronunciadas de transaminases hepáticas (TGO >4000 U/L / TGP>6000 U/L) e LDH (36000 mg/dL), além de plaquetopenia $\left(75000 / \mathrm{mm}^{3}\right)$. Nesse sentido, foi diagnosticada como Síndrome HELLP complicada.

À realização de TC de abdome evidenciou extensa área de isquemia hepática e ruptura da cápsula hepática (Figura 2). Apesar da gravidade do quadro, a paciente manteve estabilidade hemodinâmica, sem necessidade de uso de aminas vasoativas. Dessa forma, foi optado por tratamento conservador e prescrito à paciente pulsoterapia com metilprednisolona e plasmaférese após correção do RNI. Após 7 sessões de plasmaférese, paciente exibiu decréscimo progressivo de transaminases (TGO $90 \mathrm{U} / \mathrm{L} / \mathrm{TGP} 122 \mathrm{U} / \mathrm{L}$ ), LDH (230 $\mathrm{mg} / \mathrm{dL})$, restabeleceu valores de hemoglobinas $(10,2 \mathrm{mg} / \mathrm{dL})$ e plaquetas $\left(376000 / \mathrm{mm}^{3}\right)$. Portanto, um mês após a admissão no serviço, recebeu alta, estável e clinicamente melhor.

Figura 1 - Tomografia computadorizada do abdome da paciente descrita no caso - imagem real. A seta verde presente na imagem indica o hematoma hepático.

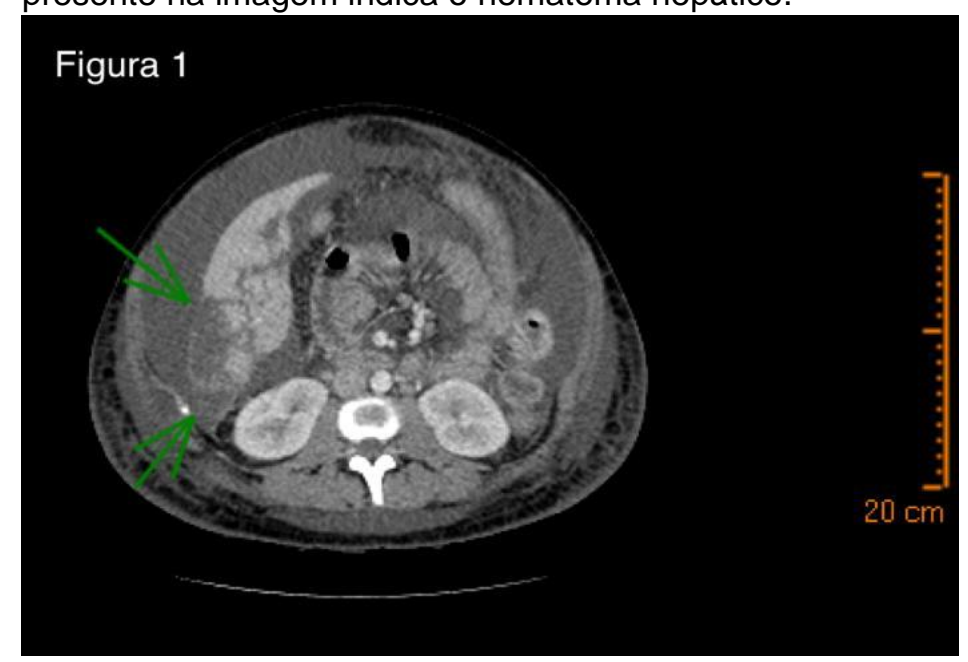

Fonte: Ribeiro VT, et al., 2020. 
Figura 2 - Tomografia computadorizada do abdomen e da pelve da paciente descrita no caso - imagem real. A seta verde presente na imagem indica a ruptura da cápsula hepática.

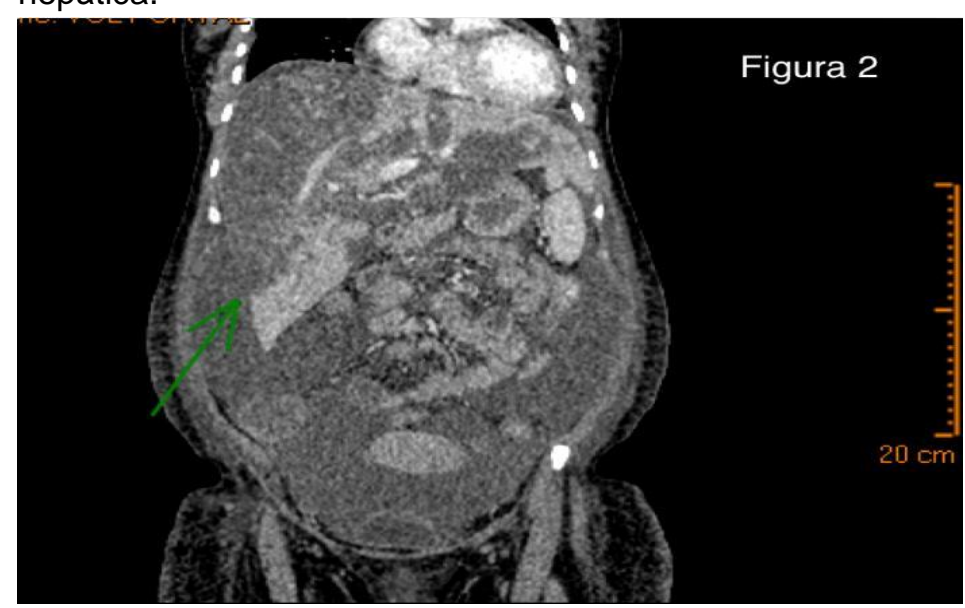

Fonte: Ribeiro VT, et al., 2020.

\section{DISCUSSÃO}

Muito se discute sobre o tratamento da síndrome HELLP não complicada, mas no caso de complicações, como o hematoma e a rotura hepática, o conteúdo encontrado na literatura é bastante limitado. A maior parte dos trabalhos disponíveis são relatos de casos, de modo que poucos estudos de grande impacto foram publicados. Nesse sentido, pelo baixo nível de evidência disponível, não há um consenso sobre qual modalidade de tratamento é superior e deve ser instituída na ocorrência de síndrome HELLP complicada (BICALHO DM, et al., 2011). Contudo, a literatura evidencia que a abordagem terapêutica está pautada em dois pilares fundamentais: clínico e cirúrgico, sendo sua indicação variada de acordo com o quadro e a gravidade da paciente em questão.

O tratamento cirúrgico é recomendado em casos de hematoma roto com instabilidade hemodinâmica, aumento do tamanho do hematoma e grande perda de volume sanguíneo (POGGIALI PVB, et al., 2010; HENRÍQUEZ-VILLASECA MP, et al., 2018). Esse manejo tem proporcionado uma diminuição da morbidade e mortalidade na síndrome HELLP com hematoma e rotura hepática, representando uma taxa de $33 \%$ de mortalidade materna no tratamento cirúrgico, comparado a $96 \%$ de mortalidade no tratamento conservador e não cirúrgico (POGGIALI PVB, et al., 2010; HUNTER SK, et al., 1995). O principal objetivo dessa modalidade de tratamento é o tamponamento do sangramento hepático (POGGIALI PVB, et al., 2010).

As pacientes com estado hemodinâmico instável deverão ser submetidas, se possível, a cesariana de urgência, seguida de laparotomia exploratória para a identificação do sangramento (FREITAS ACT, et al., 2009; BARROS BC e REZENDE MB, 2019). Durante a laparotomia, o tamponamento pode ser realizado por embalagem com compressas, uso de malhas de propileno ou malhas sintéticas absorvíveis, pontos hemostáticos no parênquima hepático, omentoplastia e uso de produtos hemostáticos (HENRíQUEZVILLASECA MP, et al., 2018).

Em casos de hemorragia não controlada, pode-se realizar a ligadura da artéria hepática (FREITAS ACT, et al., 2009). Diferentemente, quando a paciente se encontra estável hemodinamicamente, é possível a realização de arteriografia e embolização do ramo arterial sangrante. Dessa forma, o transplante hepático é o último recurso cirúrgico a ser considerado, sendo realizado quando: a hepatectomia e a anastomose portocava não foram suficientes para controlar a hemorragia; quando ocorre uma necrose hepática extensa e consequentemente, insuficiência hepática progressiva (FREITAS ACT, et al., 2009).

Não há consenso quanto ao tratamento da síndrome HELLP antes de 34 semanas de gestação, nos casos em que as condições maternas e fetais são estáveis. Dessa forma, ainda não está claro se uma postura ativa 
deve ser priorizada em relação à conduta expectante. Nesse contexto, o uso da plasmaférese pode apresentar benefícios e reduzir a morbimortalidade materna e fetal (FREITAS ACT, et al., 2009).

A plasmaférese, desenvolvida na metade do século XX, é um método terapêutico invasivo utilizado para separar o plasma da porção celular do sangue através de um circuito extracorpóreo (CEC). Quando utilizada como modelo de tratamento para alguma condição, dá-se o nome de Troca Plasmática Terapêutica (TPT), ou "Therapeutic Plasma Exchange". Seu mecanismo consiste na remoção seletiva de células ou substâncias do sangue que possam ser responsáveis por determinadas patologias, como autoanticorpos, imunocomplexos, citocinas ou outras macromoléculas; ou mesmo infundir células ou constituintes do plasma que possam reverter ou amenizar o processo patogênico, sendo então a TPT considerada uma das terapias mais eficazes em depurar o sangue e repor componentes do plasma (COHEN S e FREEMAN T, 1960).

Com relação à Síndrome HELLP, destaca-se que as alterações no organismo materno apresentam como principal fisiopatogenia uma microangiopatia trombótica gerada, possivelmente, por uma invasão inadequada do citotrofoblasto resultando em uma redução da pressão de perfusão útero-placenta. A isquemia placentária promove a liberação de citocinas, mediadores inflamatórios, fatores antiangiogênicos, espécies reativas de oxigênio e outras substâncias na circulação materna. Sendo assim, a TPT é uma estratégia válida de tratamento da Síndrome uma vez que é considerada uma das terapias mais eficazes em depurar o sangue e repor componentes do plasma. Além de ser capaz de remover toxinas endógenas como produtos decorrentes da necrose celular, a TPT remove macromoléculas combinadas com proteínas plasmáticas e imunocomplexos circulantes, e suplementa albumina plasmática, opsoninas, imunoglobulinas, e outras substâncias bioativas gerando um efeito curativo. Somado à isso, é capaz de remover substâncias e matéria prima necessárias à regeneração dos hepatócitos, o que pode afetar a recuperação hepática e a melhora da função do órgão à longo prazo (LI M, et al., 2016).

Segundo Vafaeimanesh J, et al. (2014), a plasmaférese deve ser uma opção aventada pelos médicos em situações especiais envolvendo síndrome HELLP. O autor sugere o tratamento para casos de: Síndrome HELLP incomum ou com baixa resposta ao tratamento - elevação progressiva da bilirrubina ou creatinina, trombocitopenia constante $<30000$ ou LDH $>1000$ IU / L - por mais de 72 horas após o parto; síndrome HELLP Severa; envolvimento do sistema nervoso central; síndrome HELLP associada à coagulação intravascular disseminada, bem como à disfunção de vários órgãos ou a complicações cardiopulmonares. Vafaeimanesh indica a plasmaférese também quando há diferenciação entre a síndrome HELLP e outras microangiopatias trombóticas é impossível; quando a doença é progressiva durante o parto ou após e persistente apesar do uso de hemoderivados. Considerando, então que a paciente do caso exposto manteve estabilidade hemodinâmica, e se encaixava nas indicações para TPT, o manejo conservador (não cirúrgico) foi optado em detrimento do cirúrgico.

No que tange ao benefício do uso de corticosteróides no tratamento da síndrome HELLP ainda não há um consenso estabelecido na literatura. Acredita-se que sua administração possa prevenir a queda das plaquetas e a hemólise por meio da estabilização do endotélio vascular, reduzindo o tempo de hospitalização e necessidade de hemotransfusão (MAO M e CHEN C, 2015). Por um lado, dois grandes ensaios clínicos randomizados, duplo cegos e controlados por placebo não estabeleceram benefício ao avaliarem o uso de dexametasona para melhora do desfecho em pacientes com síndrome HELLP (FONSECA JE, et al., 2005; KATZ L, et al., 2008).

Em contraponto, pequenos estudos observacionais e randomizados sugeriram que $\mathrm{O}$ uso de dexametasona acelera a resposta de recuperação dos parâmetros laboratoriais e clínicos maternos. (ISLER CM, et al., 2001; MARTIN JN, et al., 2003; O'BRIEN JM, et al., 2002). Nesse contexto, em uma meta-análise de 11 estudos $(n=550$ mulheres) comparou-se o tratamento de corticosteróides com placebo / nenhum tratamento em mulheres com síndrome HELLP. A conclusão do estudo constatou que a administração de esteróides não demonstrou uma redução significativa sobre a morte materna (razão de risco [RR] 0,95, 95\% IC 0,28-3,21), morte materna ou morbidade materna grave (RR 0,27, IC 95\% 0,03-2,12) ou morte perinatal/infantil (RR 0,64, IC 95\% 0,21-1,97), mas a diferença média na contagem de plaquetas favoreceu 0 grupo que usou corticoesteróides $(0,67$, IC 95\% 0,26-1,10) (WOUDSTRA DM, et al., 2010).

REAS/EJCH | Vol.12(10) | e5041 | DOI: https://doi.org/10.25248/reas.e5041.2020 Página 5 de $\mathbf{7}$ 
Finalmente, ressalta-se que a identificação de fatores prognósticos maternos e fetais específicos permitiria uma melhor estratificação das mulheres com síndrome HELLP, de acordo com o potencial progressivo da doença, resultando em um manejo mais individualizado. Entretanto, como já mencionado anteriormente, esses fatores ainda não são muito bem compreendidos, o que aponta para a necessidade de estudos adicionais.

O presente estudo descreveu o caso de uma paciente diagnosticada com Síndrome HELLP associada a rotura hepática espontânea, cujos resultados após abordagem conservadora foram positivos. A associação dos parâmetros clínicos, laboratoriais e de imagem possibilitam o diagnóstico e manejo precoce das complicações da Síndrome HELLP, promovendo a redução dos índices de mortalidade materna. É notória a relevância do mantenimento de uma estrita vigilância clínico-laboratorial das pacientes, haja vista a potencial gravidade do quadro. Ademais, ressalta-se que apesar da escassez de estudos referentes a Síndrome HELLP complicada por rotura hepática, o tratamento não cirúrgico pode ser benéfico na condução dessas pacientes, proporcionando desfechos favoráveis quando bem indicado; pode-se mencionar ainda que o tratamento conservador é uma abordagem menos invasiva. Contudo, mostra-se necessário o desenvolvimento de mais estudos no assunto, permitindo uma melhor compreensão dos critérios clínicos e laboratoriais que interferem no prognóstico e evolução de pacientes com síndrome HELLP. Nesse sentido, seria possível obter maior precisão nas indicações de tratamento cirúrgico ou conservador, corroborando para um melhor desfecho do quadro dessas pacientes.

\section{REFERÊNCIAS}

1. BARROS BC, REZENDE MB. Hellp síndrome ocasionando ruptura hepática: relato de caso. Revista Ciências em Saúde, 2019; 9(1):15-20.

2. BICALHO DM, et al. Hematoma hepático subcapsular no puerpério imediato. Revista Médica de Minas Gerais, 2011; 21(6):130-132.

3. BRILHANTE AVM, et al. Ruptura de hematoma subcapsular hepático como complicação da síndrome HELLP: revisão de literatura. Femina, 2010; 38(7):341-344.

4. COHEN S, FREEMAN T. Metabolic heterogeneity of human y-globulin. Biochemical Journal,1960 76(3):475-487.

5. FONSECA JE, et al. Dexamethasone treatment does not improve the outcome of women with HELLP syndrome: a double-blind, placebo-controlled, randomized clinical trial. American Journal Of Obstetrics And Gynecology, 2005; 193(5):1591-1598.

6. FREITAS ACT, et al. Rotura hepática espontânea na síndrome HELLP. Arquivos Brasileiros de Cirurgia Digestiva, 2009; 22(3):179-180.

7. HENRÍQUEZ-VILLASECA MP, et al. Hematoma subcapsular hepático roto en síndrome HELLP. Revista médica de Chile, 2018; 146 (6):753-761.

8. HUNTER SK, et al. Liver transplant after massive spontaneous hepatic rupture in pregnancy complicated by preeclampsia. Obstetrics \& Gynecology, 1995; 85(5):819-822.

9. ISLER CM, et al. A prospective, randomized trial comparing the efficacy of dexamethasone and betamethasone for the treatment of antepartum HELLP (hemolysis, elevated liver enzymes, and low platelet count) syndrome. Am $\mathrm{J}$ Obstet Gynecol, 2001; 184(7): 1332-1339.

10. KATZ L, et al. Postpartum dexamethasone for women with hemolysis, elevated liver enzymes, and low platelets (HELLP) syndrome: a double-blind, placebo-controlled, randomized clinical trial. American Journal Of Obstetrics And Gynecology, 2008; 198(3):283.1-283.8.

11. LI M, et al. Part of plasmapheresis with plasma filtration adsorption combined with continuous hemodiafiltration in the treatment of severe acute liver failure. Experimental and Therapeutic Medicine, 2016; 12(4):2582-2584.

12. MACHADO LG, et al. Rotura hepática como complicação de pré-eclâmpsia grave: relato de caso. Revista Médica de Minas Gerais, 2010; 20(4):7-10.

13. MAEJI IL, et al. Rotura hepática espontânea e síndrome HELLP: relato de caso. International Journal of Health Management Review, 2019; 5(2).

14. MAO M, CHEN C. Corticosteroid Therapy for Management of Hemolysis, Elevated Liver Enzymes, and Low Platelet Count (HELLP) Syndrome: A Meta-Analysis. Medical Science Monitor: International Medical Journal of Experimental and Clinical Research, 2015; 21:3777-3783.

15. MARTIN JN, et al. Maternal benefit of high-dose intravenous corticosteroid therapy for HELLP syndrome. American Journal of Obstetrics and Gynecology, 2003;189(3):830-834.

16. O'BRIEN JM, et al. Maternal benefit of corticosteroid therapy in patients with HELLP (hemolysis, elevated liver enzymes, and low platelet count) syndrome: impact on the rate of regional anesthesia. American Journal Of Obstetrics And Gynecology, 2002; 186(3):475-479.

17. POGGIALI PVB, et al. Rotura hepática espontânea na gestação. Revista Médica de Minas Gerais, 2010; 20(4):7-10. 
18. POLLO-FLORES $P$, et al. Alterações hepáticas da gravidez. Femina, 2015; 43(5):225-234.

19. TROJA A, et al. Management of Spontaneous Hepatic Rupture on Top of HELLP Syndrome: case report and review of the literature. Visceral Medicine, 2015; 31(3):205-208.

20. VAFAEIMANESH J, et al. Plasmapheresis: Lifesaving treatment in severe cases of HELLP syndrome. Caspian J Intern Med, 2014; 5(4):243-247.

21. WOUDSTRA DM, et al. Corticosteroids for HELLP (hemolysis, elevated liver enzymes, low platelets) syndrome in pregnancy. Cochrane Database Syst Rev, 2010; (9):CD008148. 